\title{
Aspectos médico legales y quirúrgicos de los oblitos. Presentación de un caso
}

\section{Legal and surgical medical aspects of the oblits. Presentation of a case}

Edmundo Nicasio Chóez Chiliquinga. ', María Fernanda Zambrano Pérez. ${ }^{2}$, Mayra Viviana Barros Carvajal. ${ }^{3}$, Mariana de Jesús Chuquirima Lima. ${ }^{4} \&$ Doris de la Nube Calle Mendoza. ${ }^{5}$

\begin{abstract}
.
Introduction: The oblite is a foreign body forgotten inside a patient during a surgical intervention; its real incidence is not clear because in many cases they are not reported in the hospital because of the medical-legal implications. Case report: A 41-year-old male patient goes to the emergency department for presenting a complicated appendicitis chart, an emergency surgery is

\section{Resumen.}

Introducción: El oblito es un cuerpo extraño olvidado en el interior de un paciente durante una intervención quirúrgica, su incidencia real no es clara pues en muchas ocasiones no se reportan en el medio hospitalario por las implicaciones médico-legales. Informe de caso: Paciente masculino de 41 años de edad, acude al servicio de emergencia por presentar cuadro de apendicitis

1 Especialista en Medicina Forense, Universidad Central del Ecuador, Quito, Ecuador. edmundo.choez@gmail.com, (D) https://orcid.org/0000-0002-4036-8497

2 Especialista en Medicina Forense, Universidad Central del Ecuador, Quito, Ecuador. a15_fernanda@hotmail.com, (D) https://orcid.org/0000-0003-1947-8437

3 Especialista en Medicina Forense, Universidad Central del Ecuador, Quito, Ecuador. mvbcmedi7@hotmail.com, (D) https://orcid.org/0000-0003-2932-2153

4 Especialista en Medicina Forense, Universidad Central del Ecuador, Quito, Ecuador. marianachuquirima@gmail.com, (D) https://orcid.org/0000-0002-4701-4907

5 Especialista en Medicina Forense, Universidad Central del Ecuador, Quito, Ecuador. churona123@hotmail.com (iD) https://orcid.org/0000-0002-2057-1383
\end{abstract}


performed, subsequently presents an enterocutaneous fistula. Patient goes to the doctor which evidences the presence of a foreign body at the level of the wound, which is extracted. Discussion: When there are dark diagnostic pictures after surgery, the clinical suspicion of an oblite should be followed by the completion of complementary examinations and immediate removal. Conclusion: Understanding this complication will allow adequate preventive measures to be taken and will facilitate prompt clinical suspicion with timely diagnosis and appropriate treatment.

Keywords: Oblito, Responsibility, Medicine, Legal, Prevention. complicada, se realiza una intervención quirúrgica de emergencia, posteriormente presenta una fístula enterocutánea. Paciente acude a facultativo el cual evidencia a nivel de la herida presencia de cuerpo extraño, el cual es extraído. Discusión: Cuando existen cuadros diagnósticos oscuros posterior a una intervención quirúrgica la sospecha clínica de un oblito se la debe hacer seguido de la realización de los exámenes complementarios y extracción inmediata. Conclusión: La comprensión de esta complicación permitirá tomar medidas preventivas adecuadas $\mathrm{y}$ facilitará una pronta sospecha clínica con un diagnóstico oportuno y tratamiento adecuado.

\begin{tabular}{|c|c|}
\hline Palabras & claves: \\
\hline Responsabilidad, & Medicina, \\
\hline
\end{tabular}

\section{Introducción.}

La palabra "oblito", deriva del latín "oblitum-oblivus" olvidado-olvido; se lo usa para definir a todo cuerpo extraño olvidado dentro del cuerpo de un paciente, durante el curso de un procedimiento quirúrgico (Lupi, José; Divito, José; Leoncio, 2003).

Se presentan aquellos sinónimos utilizados en la literatura médica: "compresomas", "pseudotumor de gasa”, "gossypibomas” (término anglosajón) "gasomas", "oblitomas", "oblitos quirúrgicos", "cuerpos extraños", "ensuciado", "emporcado", “corpus allienum", "textilomas", resultando este último el más usado en la actualidad para definir este "accidente" con un término no médico. No obstante ello, y salvo cuestiones estrictamente puntuales, es claro el sentido genérico que todos le asignan en estos casos, a los "cuerpos extraños" de cualquier naturaleza, olvidados durante la cirugía. En contraposición a lo que sucede con otros objetos como clips metálicos o prótesis que son colocados intencionalmente con mucho mayor frecuencia (María et al., 2017).

En la actualidad el vocablo oblito, ya está incluido en el diccionario de la Real Academia Española, y definido como cuerpo extraño olvidado en el interior de un paciente durante una intervención quirúrgica (Española, 2017). 
$\mathrm{Su}$ incidencia oscila entre 1 por cada 8.000 a 18.000 intervenciones quirúrgicas, la frecuencia reportada internacionalmente es de 1 por cada 1500 laparotomías solo para las cirugías abdominales; otras comunicaciones exponen una ocurrencia que alcanza 1 por cada 3000 entre todas las intervenciones quirúrgicas (Chinelli, Javier; Olivera, Eduardo; Rodríguez, 2016).

La verdadera frecuencia de este evento adverso es difícil de establecer ya que, por una parte, los enfermos pueden permanecer asintomáticos por tiempo indefinido, a los que se les diagnóstica y resuelve el problema quedan como casos aislados sin registro epidemiológico del evento adverso quirúrgico y, por otra parte, los casos pueden ser ocultados por el mismo personal hospitalario para evitar una demanda médico legal por lo que existe un importante subregistro del evento (Murguía, Paniagua, Rivera, \& Ruiz, 2011).

Es de vital importancia la autopsia hospitalaria en pacientes fallecidos en un postoperatorio inmediato, el hallazgo casual o clínicamente sospechada, de algún oblito (Lupi, José; Divito, José; Leoncio, 2003).

Los síntomas son inespecíficos y pueden aparentar diversos padecimientos dependiendo de la región que se encuentre comprometida y el tiempo que transcurra desde el procedimiento quirúrgico. Existen dos tipos de respuestas al cuerpo extraño, aguda y crónica; la primera es una reacción inflamatoria exudativa con la formación de absceso y fístula, la segunda es una reacción fibrótica aséptica con posibles complicaciones futuras. Se ha reportado que puede pasar desde algunos días del postoperatorio hasta varias décadas en ser diagnosticado (Silva-carmona, Ganado-escobar, \& Mondragónchimal, 2014).

Por tanto se debe sospechar la presencia de un cuerpo extraño en todo aquel paciente que desarrolle una masa ocupativa, una lesión tumoral, alguna fístula o trayecto fistuloso, o salida de alguna secreción aséptica de no estar infectada o contaminada; que ésta sea persistente a pesar de los tratamientos conservadores aplicados o bien que se presenten cuadros de obstrucción intestinal recientes a alguna cirugía (Manrique, Jorge; Pelish, Andrés; Marino, Marcelo; Mrad, José; Kahné, 2003).

De los oblitos entre los más frecuentes se encuentran las gasas, campos y otros materiales utilizados para enjugar o empaquetar vísceras en el campo operatorio. En segundo lugar, se ubican los instrumentos quirúrgicos o restos de los mismos como agujas, hojas de bisturí, pinzas, cánulas o trócares. Como miscelánea, se citan objetos de uso personal de miembros del equipo quirúrgico como bisutería y anteojos (Manrique, Jorge; Pelish, Andrés; Marino, Marcelo;Mrad, José; Kahné, 2003).

Aproximadamente un $50 \%$ del material quirúrgico retenido son descubiertos por lo menos a los 5 años de la cirugía y hay reportes en la literatura de hasta 30 años después del evento quirúrgico (Alberto et al., 2009). 
Se divisa mayor predominio en el sexo femenino en el $57 \%$ con mayor frecuencia en las cirugías del sistema digestivo, seguidas por las cirugías ginecológicas, urológicas, vasculares, de tórax y por trauma. Los oblitos no son exclusivos del abdomen y pueden estar presentes en el muslo, donde causan edema, dolor, reacción perióstica y aumento de vasos sanguíneos, o aparentan lesiones tumorales, en el tórax, donde producen dolor localizado y tos persistente no hemoptoica (Miranda \& Aguilar, 2011).

El diagnóstico inicial en el $90 \%$ de los pacientes se realiza mediante una radiografía simple de abdomen en dos proyecciones, mientras que en el $10 \%$ restante requerirá de tomografía computada, ultrasonido o resonancia magnética (Córdova-pluma et al., 2012).

El hallazgo de un oblito puede ser sencillo en los casos en que se usan compresas marcadas con material radiopaco el cual se detecta en las radiografías simples de abdomen. Excepcionalmente puede verse en estas radiografías simples imágenes en "panal de abejas", "enrejillado" o en "miga de pan", que coincide con la compresa infiltrada por secreciones y gas, luego de micro perforaciones en la pared intestinal, también puede ser mal interpretada con la imagen de por presencia de heces, pero su ubicación fuera del colon y su constancia descartan esta eventualidad. Las series gastrointestinales con contraste, en los enfermos no ocluidos, son de valor al mostrar el defecto de lleno intraluminal y en ocasiones puede penetrar el contraste en la cavidad del absceso a través del cual, previa fistulización, migró la compresa a la luz intestinal. La ecografía es de gran valor al observar una estructura compleja que produce sombra acústica y varia paralelo a la dirección del transductor. Exámenes complementarios como la resonancia magnética y la tomografía axial computarizada son imprescindibles para su identificación, en el caso de textilomas agudos se visualiza una masa heterogénea que contiene gran cantidad de aire atrapado pudiendo tener una anillo hiperdenso periférico, mientras que en los textilomas crónicos se parece a un tumor que no capta el contraste endovenoso, con calcificaciones en su interior e impresionan una masa sólida con poco o ningún aire atrapado en su interior (Silva-carmona et al., 2014).

El tratamiento es la extracción quirúrgica, cuando se realiza antes de los 15 días, extracción precoz es técnicamente fácil y tiene mejores resultados. En casos crónicos se produce un proceso adherencial que involucra órganos vecinos, convirtiendo la cirugía más compleja que puede requerir incluso resecciones viscerales, las cuales se asocian con una morbilidad y mortalidad mayores (Campos-castolo, Hernández-gamboa, Victoria-ochoa, Alejandría, \& Vega, 2008).

\section{Reporte de caso}

Paciente de sexo masculino de 41 años de edad, acude al servicio de emergencia por presentar cuadro clínico compatible con abdomen agudo inflamatorio (apendicitis) se le realizan exámenes complementarios y se cataloga como apendicitis complicada por lo que se realiza una intervención quirúrgica de emergencia, se realiza laparotomía media infraumbilical más apendicetomía, más lavado de cavidad, más colocación de drenaje 
por apendicitis grado IV más plastrón y absceso localizado. No se reportan accidentes trans-operatorios y con un "conteo de compresas completo", según consta en el Informe Operatorio, se decide finalizar la operación. Durante el postquirúrgico, el paciente presentó dolor abdominal, vómito y alzas térmicas, sin embargo no le realizaron exámenes complementarios de imagen como radiografía simple de abdomen o tomografía para atribuir una causa a su cuadro clínico. Paciente es dado de alta y control por consulta externa. Por el hecho de haber sido una cirugía contaminada paciente presenta infección de sitio quirúrgico y presencia de fístula enterocutánea, además es hospitalizado por segunda ocasión donde se le realiza una tomografía simple y contrastada de abdomen en donde se reporta un artefacto con densidad de metal en mesenterio, sin embargo aparentemente nadie se percató de esto y el paciente es dado de alta. Paciente acude a servicio médico particular donde cirujano valora y extrae funda de colostomía y se evidencia a nivel de la herida abundante secreción purulenta con presencia de cuerpo extraño, el cual es extraído.

El paciente presentó una evolución postquirúrgica tórpida con varios ingresos a hospitalización que requirieron antibioticoterapia, curaciones y tratamientos prolongados, todo esto probablemente a la presencia de un oblito (cuerpo extraño) olvidado en su cavidad abdominal. Una vez realizado el análisis de las historias clínicas de los establecimientos involucrados en el proceso de atención del paciente y entrevistas realizadas tanto al paciente como a los profesionales de salud aparentemente si presentó un cuerpo extraño (compresa quirúrgica) en su cavidad abdominal a la intervención quirúrgica que le realizaron en primera instancia.

\section{Discusión}

El concepto de cirugía segura implica la certeza de que se estará libre de riesgo, por ejemplo, de daño o eventos adversos, lo cual es prácticamente imposible, ya que todo acto quirúrgico implica asumir riesgos. La presencia de material quirúrgico retenido en forma no intencional es un evento adverso potencialmente evitable (Chinelli, Javier; Olivera, Eduardo; Rodríguez, 2016).

Este tipo de problema puede ocurrir incluso en aquellos cirujanos experimentados y cautos. Sin embargo para el común de la gente, el tomar conocimiento de la existencia de un oblito y la casi segura reintervención consecuente para solucionarlo; no deja de ser una circunstancia que siempre va a llevar implícita una actitud negligente del personal involucrado, lo que conllevaría a sanciones legales y además de la reparación del afectado (Lupi, José; Divito, José; Leoncio, 2003).

Los oblitos al ser parte de un evento quirúrgico son considerados como una iatrogenia, que pueden ocasionar la muerte de los individuos que padecen dicha patología. El estudio de los oblitos tiene intervenciones éticas y legales. Al realizar una cirugía existe un equipo de trabajo y los encargados directamente del conteo del material son el circulante y la enfermera que instrumenta, sin embargo, al existir algún error la responsabilidad recae hacia todo el equipo (Campos-castolo et al., 2008). 
La palabra iatrogenia tiene un origen griego y se traduce como inducido por el sanador o médico. Se ha considerado como iatrogénica la condición de la identificación de un cuerpo extraño dentro del cuerpo posterior a algún procedimiento quirúrgico, sin que ello sea atribuible a mala intención, conocimientos, destrezas y sí al error humano (Alberto et al., 2009).

La falta de éxito o la aparición de complicaciones a raíz de un tratamiento médico, no implica de por si una hipótesis de responsabilidad médica. Para que exista responsabilidad médica o mala praxis se requiere la concurrencia de antijuridicidad, daño, factores de imputación de la responsabilidad y la relación de causalidad entre conducta y consecuencia dañosa. La ley tiene una postura crítica frente al oblito. Lo atribuye a una actitud negligente que reúne factores como para catalogarlo de lesión culposa y por tanto merecedora de sanción. Todo ser humano es responsable de sus actos y de las consecuencias que ocasione en ese sentido debe reparar el daño por sí solo ya sea voluntario o no. Se culpabiliza al médico como garante del control y fiscalización del equipo humano que lo acompaña; resulta solidario con ellos en la responsabilidad al igual que a la entidad donde se efectúa la intervención. El cirujano puede disminuir su responsabilidad invocando y demostrando que el olvido se produjo pese a haber mantenido una conducta idónea, prudente y diligente, destinada a evitarlo. En el seguimiento posquirúrgico se debe acreditar la aplicación oportuna de los métodos dirigidos a detectar la presencia y corregir las consecuencias del hecho. La evaluación del comportamiento galénico requiere la adecuada consideración de las "las circunstancias de las personas, del tiempo y del lugar", vale decir la urgencia de la indicación quirúrgica, las circunstancias en que se realizó, las condiciones y el estado del paciente, la complejidad del acto operatorio, el tipo y características del material olvidado, entre otros aspectos. Asumir complicaciones no es agradable y las circunstancias son diferentes cuando se trata de aceptar el error propio o denunciar el fallo cometido por otro. El paciente tiene derecho de conocer la verdad. El médico debiera honrarlo e informar al paciente del diagnóstico. Tanto el paciente como el médico merecen respeto y cuidado. Nadie puede ser obligado a autoinculparse ni a denunciar a un tercero. Si se informa del oblito, se debe especificar que es una complicación propia de los procedimientos invasivos y que su ocurrencia es posible. La decisión caso por caso es propia de la toma de decisiones en medicina y coherente con la bioética. La conducta por adoptar, forma parte del actuar privado y depende de cada uno. Cuando supere la capacidad de decisión del médico, corresponde consultar con el Comité de Ética Hospitalaria, asesores legales institucionales y/o personales (Manrique, Jorge; Pelish, Andrés; Marino, Marcelo;Mrad, José;Kahné, 2003).

Existen diferentes términos como son las impericia, negligencia, imprudencia en ese sentido vamos analizar que término corresponde a este tipo de problema. La impericia en oblitos, debe ser descartada. La imprudencia, puede ser adecuada en forma individual; es poco prudente en este tipo de sucesos en procedimientos quirúrgicos. El caso puntual de los oblitos, constituye parte de la negligencia, dentro del cuadro 
conductual culposo por parte del personal de salud (Lupi, José; Divito, José; Leoncio, 2003).

En nuestra legislación en relación a la práctica profesional en el Código Orgánico Integral Penal Registro Oficial Suplemento 180 de 10-feb-2014 se menciona lo siguiente:

Lo relacionado con mala práctica profesional y como resultado fallece la persona esta descrito en el artículo 146. En cambio si la persona sobrevive se tomará en cuenta lo descrito en el artículo 152 (A. N. del Ecuador, 2014).

En cambio en La ley Orgánica de Salud Registro Oficial Suplemento 423 de 22-dic2006 señala lo siguiente:

En el artículo 202 indica que constituye infracción en el ejercicio de las profesiones de salud, todo acto individual e intransferible, no justificado, que genere daño en el paciente y sea resultado de: a) Inobservancia, en el cumplimiento de las normas; b) Impericia, en la actuación del profesional de la salud con falta total o parcial de conocimientos técnicos o experiencia; c) Imprudencia, en la actuación del profesional de la salud con omisión del cuidado o diligencia exigible; y, d) Negligencia, en la actuación del profesional de la salud con omisión o demora injustificada en su obligación profesional (C. N. del Ecuador, 2012).

Al oblito se lo puede valorar como un incidente desafortunado. Incidente, porque es realmente un evento impensado que ocurre en el desarrollo de un asunto y tiene con éste algún enlace. Desafortunado, porque no es únicamente resultado de la buena suerte, que provoque felicidad, ni resultado de ella (Lupi, José; Divito, José; Leoncio, 2003).

Más que transformar tal incidente desafortunado en un hecho legal, con claros fines financieros y sin tener en cuenta la probabilidad del error humano involuntario, el razonamiento más lógico seguiría un estudio de las causas que propiciaron el hecho para su posterior prevención. Entre los factores que más frecuentemente se relacionan con la incidencia de cuerpos extraños olvidados en las cavidades corporales están: cirugías de emergencia con uso de gran cantidad de compresas y cambio de personal auxiliar transoperatorio, cirugías en condiciones de estrés como en guerras o recepciones masivas de heridos, casos complejos, cirugías nocturnas con equipo quirúrgico agotado (Silva-carmona et al., 2014).

De acuerdo a un meta análisis se identificaron algunos factores de riesgo para que se presentan en los oblitos, estos son: falta de recuento de material quirúrgico o fallas en la realización del mismo, cirugía prolongada, realización de más de un procedimiento, ocurrencia de eventos imprevistos, sangrado mayor a quinientos mililitros (Moffattbruce, Cook, Steinberg, \& Stawicki, 2014). 
Al-Qurayshi et al, en un estudio caso control concluye que también la edad avanzada, la obesidad y los hospitales docentes, se comportan como factores de riesgo que aumentarían la incidencia de casos (Al-qurayshi, Hauch, \& Slakey, 2015).

Se ha determinado que el conteo tiene una sensibilidad del $77 \%$ y especificidad del $99 \%$, lo cual demuestra claramente la posibilidad de error. Cuando existe discrepancia del conteo o la cuenta está incompleta, la posibilidad de oblito se incrementa por lo que debe realizarse un estudio radiológico de la cavidad quirúrgica para eliminar la posibilidad (Murguía et al., 2011).

Existen diferentes mecanismos para evitar que se presenten los oblitos como son: el uso de marcadores que sean radiopacos en el material que se vaya a utilizar, otro de los puntos relevantes es contar todo el material usado durante la cirugía, en este sentido se recomienda que el conteo sea por tres ocasiones al abrir el material, previo a cerrar la cavidad y al final del cierre (Chinelli, Javier; Olivera, Eduardo; Rodríguez, 2016).

En los Estados Unidos en un centro hospitalario se implementó el sistema de gasa segura, el cual, está aprobado por entidades internacionales como es la FDA, este sistema permite el conteo de gasas a través de un código de barras y se lo realiza antes y al final de la cirugía (Alberto et al., 2009).

\section{Conclusiones.}

- La comprensión de esta complicación permitirá tomar medidas preventivas adecuadas y facilitará una pronta sospecha clínica con un diagnóstico oportuno y tratamiento adecuado. Los oblitos generan problemas médico legales en cuanto a responsabilidad profesional tanto a nivel civil como penal. Dentro del esquema conductal culposo; se ha considerado como negligente la actitud del médico que deja un oblito.

- Existen algunas situaciones de emergencia quirúrgica, en las que esta complicación debería justificarse como un error inculpable, por parte de las autoridades judiciales. Entre las medidas para su prevención están la realización del recuento de material quirúrgico, así como de métodos auxiliares que pueden aplicarse en forma sistemática o bien ante recuentos incorrectos (radiografía intraoperatoria, rastreo de instrumental quirúrgico utilizando código de barras o radiofrecuencia, uso de compresas con marcadores radiopacos, entre otros), aunque ninguno de ellos en forma aislada o en conjunto han podido eliminar por completo el riesgo de aparición de oblitos.

- El trabajo meticuloso y responsable de todos los miembros del equipo quirúrgico resulta esencial para garantizar un buen resultado final e impedir que una intervención quirúrgica termine en este tipo de complicación, que puede ser completamente evitable. 
- Es fundamental que el protocolo de conteo de material quirúrgico adaptado por cada Institución de Salud conste en la historia clínica; puesto que puede ser valorado como punto fundamental defensivo, ante un problema judicial posterior. Es importante considerar que es necesario generar una cultura que permita que se reporten estos hechos, sin que ello implique una acción médicolegal inmediata y punitiva, estableciéndose que el personal médico se responsabilice del error y su pronta solución.

\section{Referencias bibliográficas.}

Al-qurayshi, Z. H., Hauch, A. T., \& Slakey, D. P. (2015). Retained Foreign Bodies : Risk and Outcomes at the National Level. Journal of the American College of Surgeons, 220(4), 749-759. https://doi.org/10.1016/j.jamcollsurg.2014.12.015

Alberto, G., Ramírez, M., Treviño, C. R., Luis, J., Arias, R., Eugenia, M., ... Méndez, A. (2009). Material quirúrgico retenido y acciones para evitar el error . Medigraphic, 7(3), 158-164.

Campos-castolo, D. E. M., Hernández-gamboa, L. E., Victoria-ochoa, L. E. O. R., Alejandría, L., \& Vega, V. (2008). Morbilidad derivada del olvido de gasas en actos quirúrgicos. Revista CONAMED, 13(1), 5-11.

Chinelli, Javier; Olivera, Eduardo; Rodríguez, G. (2016). Oblitos en Cirugía . Análisis de casuística y estrategias para su prevención. AnFaMed, 4(2), 47-55.

Córdova-pluma, V. H., Vega-lópez, C. A., Castillo-gonzález, F. A., Cornejo-lópez, G. B., Ramírez-arias, J. L., \& Rodríguez-treviño, C. (2012). Caso clínico Textiloma abdominal: reporte de caso. Med Int Mex, 28(4), 398-402.

Ecuador, A. N. del. (2014). Código orgánico integral penal , COIP, 1-219.

Ecuador, C. N. del. Ley organica de salud (2012).

Española, R. A. (2017). Diccionario de la lengua española, Edición del Tricentenario. Real Academia Española (RAE). Retrieved from http://dle.rae.es/?id=UV6hPaS

Lupi, José; Divito, José; Leoncio, C. (2003). Oblitos Quirúrgicos Aspectos Medico Legales y Eticos Reseña Jurisprudencial. Cuadernos de Medicina Forense, 1, 4358 .

Manrique, Jorge; Pelish, Andrés; Marino, Marcelo;Mrad, José;Kahné, M. (2003). Oblito: Incidencia, Consecuencias Médicas, Legales y Éticas. Revista Argentina de Cirugía, 85(1-2), 31-35.

María, J., Ortega, G., Ailin, I. E., Díaz, G., Est, I. I., Luis, A., .. Ii, S. (2017). Textiloma Intra-abdominal . Reporte de un caso. Rev Méd Electrón, 39, 11331142 .

Retrieved from 
http://www.revmedicaelectronica.sld.cu/index.php/rme/article/view/1775/3606

Miranda, R., \& Aguilar, W. (2011). Reporte de caso clínico : cuerpo extraño en abdomen Clinical case report: foreign body in abdomen. Rev. Med. FCM-UCSG, 16(3), 231-236.

Moffatt-bruce, S. D., Cook, C. H., Steinberg, S. M., \& Stawicki, S. P. (2014). ScienceDirect Risk factors for retained surgical items: a meta-analysis and proposed risk stratification system. Journal of Surgical Research, (June), 1-8. https://doi.org/10.1016/j.jss.2014.05.044

Murguía, E. B., Paniagua, A. H., Rivera, A. M., \& Ruiz, F. T. (2011). Retención de cuerpos extraños en cirugía: La seguridad del enfermo en riesgo. Medigraphic Cirujano General, 33(3), 175-179.

Silva-carmona, A., Ganado-escobar, A. M., \& Mondragón-chimal, M. A. (2014). Textiloma que se presenta como una masa abdominal: presentación de un caso y revisión bibliográfica. Revista de Medicina e Investigación, 2(1), 18-22.

\section{LCiencia}


PARA CITAR EL ARTÍCULO INDEXADO.

Chóez Chiliquinga, E. N., Zambrano Pérez, M. F., Barros Carvajal, M. V., Chuquirima Lima , M. de J., \& Calle Mendoza, D. de la N. (2021). Aspectos médico legales y quirúrgicos de los oblitos. Presentación de un caso . Anatomía Digital, 4(2), 142-152. https://doi.org/10.33262/anatomiadigital.v4i2.1.1695

\section{Ciencia \\ LDigital}

El artículo que se publica es de exclusiva responsabilidad de los autores y no necesariamente reflejan el pensamiento de la Revista Anatomía Digital.

El artículo queda en propiedad de la revista y, por tanto, su publicación parcial y/o total en otro medio tiene que ser autorizado por el director de la Revista Anatomía Digital.
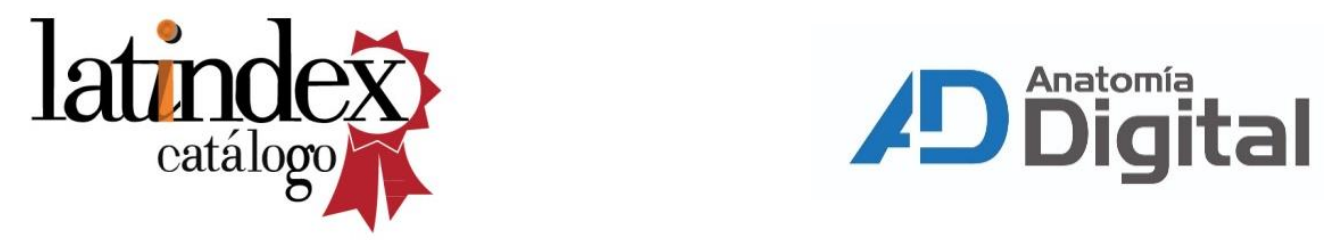